\title{
Essais
}

Revue interdisciplinaire d'Humanités

$2 \mid 2012$

Aux marges de l'humain

\section{Maxence Alcade, L'Artiste opportuniste : entre transgression et posture}

\section{Elisabeth Spettel}

\section{(2) OpenEdition}

1 Journals

Édition électronique

URL : https://journals.openedition.org/essais/10537

DOI : 10.4000/essais. 10537

ISSN : 2276-0970

Éditeur

École doctorale Montaigne Humanités

\section{Édition imprimée}

Date de publication : 15 novembre 2012

Pagination : 114-117

ISBN : 978-2-86781-857-8

ISSN : 2417-4211

\section{Référence électronique}

Elisabeth Spettel, «Maxence Alcade, L'Artiste opportuniste : entre transgression et posture », Essais [En ligne], 2 | 2012, mis en ligne le 17 janvier 2022, consulté le 20 janvier 2022. URL : http://

journals.openedition.org/essais/10537 ; DOI : https://doi.org/10.4000/essais.10537 


\section{Un chercheur, un livre Recensions}

Maxence ALCADE, L'Artiste opportuniste: entre transgression et posture, Paris, L’Harmattan, 2011

« Notre intuition est que non seulement il existe encore de nos jours des transgressions dans l'art actuel et qu'en plus il est possible de grouper ces postures au sein d'un genre ${ }^{1}$."

Dans son ouvrage L'artiste opportuniste, Maxence Alcade part d'un constat: une grande partie des œuvres modernes semble évaluée à partir de la catégorie de transgression qui devient un véritable " critère " artistique. Or, en devenant quasiment un impératif de création, la transgression finit par se vider de son sens. L'enjeu de cet ouvrage est de tenter de questionner ce concept, et, à travers lui, d'interroger l'œuvre d'art contemporaine elle-même et ses modes de production. Que signifie une ouvre transgressive? Quels sont ses rapports au pouvoir et au politique? La transgression ne se redéfinit-elle pas sans cesse par rapport au contexte, aux nouvelles lois et normes d'une société?

De nos jours, nous pouvons encore parler de formes de transgressions dans l'art actuel, différentes de celles des avant-gardes et, dans le même temps, de l'émergence de la figure de l'" artiste opportuniste». L'auteur insiste sur le fait qu'il ne faut pas voir dans cet adjectif une connotation péjorative mais un nouveau type de relation au pouvoir. Dans cette optique, son ambition est d'élaborer une typologie des transgressions dans la lignée d'Anthony Julius tout en questionnant le nouveau statut de l'artiste, entre désir de liberté et nécessité de jouer avec les "règles » du monde de l'art.

Transgresser signifie " passer outre » un interdit, une loi et peut s'établir sur trois plans qui s'imbriquent et se complètent:

- La transgression des règles de l'art: les œuvres qui procèdent de ce type de transgression proposent un travail sur la définition même de l'art.

- La transgression des tabous : un art qui a comme visée première de choquer le public mais dont les formes restent cependant assez conventionnelles.

1. Maxence Alcade, L'Artiste opportuniste: entre transgressions et postures, Paris, L'Harmattan, 2011, p. 9. 
L'auteur prend comme exemple la série The Morgue d'Andreas Serrano dont la composition et la monumentalité renvoient à une certaine tradition de représentation de la mort dans l'histoire de l'art.

- La transgression comme résistance politique qui s'oppose à l'art de propagande. Alcade compare les photomontages de Heartfield avec les images de la propagande nazie: alors que les premiers opèrent une déconstruction qui questionne notre regard, les secondes n'offrent qu'un sens évident et univoque.

Comme le souligne Paul Ardenne dans L'Art dans son moment politique, il est important de ne pas confondre pouvoir et politique. Si les frontières sont floues entre ces deux notions, le pouvoir désigne une instance dirigeante tandis que le politique renvoie à une position idéologique au sein de la cité. Ainsi, tout art qui s'attaque au pouvoir n'est pas forcément un art politique et, de la même manière, tout art qui se dit politique ne s'attaque pas nécessairement au pouvoir. Il existe même aujourd'hui une relation paradoxale voire contradictoire entre art et pouvoir.

Lauteur remarque non sans surprise que de nombreuses œuvres arborent l'étiquette d'art engagé politiquement mais sont subventionnées par l'institution qu'elles contestent par ailleurs. Qui plus est, la transgression, de critère de valeur artistique, devient facteur de cotation, alimentant la logique du marché de l'art.

À l'époque de la "subversion subventionnée ${ }^{2}$ ", l'artiste contemporain peut tomber dans la facilité d'une provocation sans cesse renouvelée qui conduit à un rejet de la part d'un public non spécialiste. Ce dernier lui reproche la disparition de repères et de critères esthétiques permettant une évaluation des ouvres d'art et la disparition d'un engagement authentique contre le pouvoir dans la lignée des avant-gardes. En effet, à la différence de Dada et du surréalisme, l'art contemporain ne s'élabore pas en marge des circuits officiels étatiques ou artistiques mais est récupéré et totalement intégré par ces derniers. Il entre dans le marché de l'art, d'où un questionnement sur la crédibilité de son message.

Cette forme de récupération " en amont " des œuvres qui relèvent souvent de commandes ${ }^{3}$, s'accompagne d'un changement du statut et de la mission des musées et centres d'art. Ces structures mènent ainsi une politique de l'événementiel répondant à une volonté de toucher un plus large public. Les récentes expositions confrontant les collections anciennes des musées à des pièces actuelles comme celles de Jeff Koons ou de Murakami à Versailles

2. Rainer Rochlitz, Subversion et subvention, Paris, Gallimard, « NRF Essais », 1994.

3. Dans le cadre des exemples traités par Maxence Alcade: Gianni Motti ou Maurizio Cattelan. Car il existe également un art subversif récupéré a posteriori comme le street art, nouvelle coqueluche des collectionneurs. 
témoignent de ce passage d'une activité de conservation historique à une préoccupation de l'immédiat, empruntant presque une logique médiatique.

Cependant, Maxence Alcade ne cherche en aucun cas à dresser un tableau manichéen avec d'un côté, les artistes avant-gardistes à l'engagement sincère luttant contre tout ordre établi et, de l'autre, les artistes contemporains complètement récupérés par le marché de l'art. La situation est beaucoup plus complexe que cela car le contexte a profondément changé. On observe un passage de l'"inscription historique " à "l'inscription dans l'instant", autrement dit à un "opportunisme de la contingence 4 ".

À partir des années 1990 avec la "fin des utopies", le rêve de "changer la vie " cher aux modernes et plus spécifiquement aux surréalistes disparaît. La notion de collectif éclate en postures "activistes" individuelles qui ne débordent pas du circuit de l'art:

Les artistes sont désormais pleinement conscients du fonctionnement du monde de l'art auquel ils participent, ils adoptent la négociation au détriment de la confrontation préconisée par leurs aînés.

Maurizio Cattelan joue le rôle de bouffon "autorisé à aiguiller le prince ${ }^{5}$ " qui flatte le pouvoir qu'il dénonce par ailleurs. Ainsi, les artistes actuels se plaisent à tordre, détourner, mettre à mal les lois du champ de l'art tout en ayant pleinement conscience des limites à ne pas dépasser.

Pour conclure, ce livre questionne toutes les ambiguïtés et les contradictions propres à l'idée d'un art transgressif, à la croisée de divers territoires, tiraillé entre le besoin d'affronter toutes formes de pouvoir et la nécessité d'exister et d'avoir une visibilité. Maxence Alcade part du monde de l'art mais est obligé de passer par le "détour » de la politique, de la sociologie, de la philosophie ou encore de la culture pour interroger la place de l'artiste et de la transgression dans la création d'aujourd'hui. Chaque œuvre est le fruit d'un travail collectif et de différents facteurs, bien loin du mythe de la création ex nibilo. Il s'agit de saisir cet entremêlement de morceaux de vie, de pensée, d'action au sein de chaque pièce et toutes les problématiques qui en surgissent.

Cette démarche s'inscrit au cœur de ma propre recherche qui tente d'analyser le "double jeu de la subversion " à travers une comparaison historique entre trois périodes: le dadaïsme, le surréalisme et enfin l'art contemporain. Cette problématique se situe aux confins de différents champs d'étude: esthétique, philosophie, histoire, sociologie et pousse à adopter un regard nuancé. La subversion désigne cette relation entre l'artiste, l'œuvre et le spectateur, en

4. Maxence Alcade, op. cit., p. 85.

5. Évelyne Toussaint, La fonction critique de l'art: dynamiques et ambiguïtés, Bruxelles, Lettre volée, 2009, p. 117. 
constante reconfiguration selon le contexte dans lequel elle s'inscrit. Loin d'apporter une réponse définitive, l'essai L'Artiste opportuniste ouvre de nombreux horizons et soulève de nombreuses questions. L'artiste ne serait-il pas ce subversif habile qui " doit casser les règles de l'art sans pour autant se faire exclure du jeu ${ }^{6} »$ ?

Elisabeth Spettel, université Michel de Montaigne Bordeaux 3, EA 4593 CLARE, elisabeth.spettel@gmail.com

\section{Tim PALMER, Brutal Intimacy: Analyzing Contemporary French Cinema, Middletown, Conn.: Wesleyan University Press, 2011, 287 p.}

Brutal intimacy, non traduit à ce jour, est le premier essai de Tim Palmer. Cet ouvrage apporte un regard original sur le cinéma français des années 2000. Cette " intimité brutale » dont parle l'auteur interroge l'évolution du concept d'intimité dans le cinéma français contemporain. En couverture, il propose un premier indice: un photogramme issu du film Irréversible de Gaspard Noé. Vincent Cassel et Monica Bellucci s'embrassent à travers le rideau de la douche et dévoilent un moment d'intimité à l'écran. La tendresse qui caractérise ce plan arrive après plusieurs séquences brutales dont un viol et un meurtre qui ont beaucoup marqué le public au moment de la sortie du film en 2002.

Tim Palmer, chercheur anglais, est Associate professor à l'université de Caroline du Nord et un fin connaisseur du cinéma français. Sans se limiter à une approche auteuriste ou seulement économique, l'auteur fait le lien entre les conditions de production et les innovations visuelles des films français produits entre 2000 et 2010. Il s'intéresse particulièrement à la façon dont les films sont présentés au public et mis en valeur par les institutions du cinéma (La FEMIS, L'Académie des Césars). Le point de vue d'un universitaire nordaméricain sur notre cinématographie nationale est précieux tant il est délicat de faire une analyse aussi objective et distanciée de l'intérieur. Son corpus très vaste, peut-être trop, va de Luc Besson à Claire Denis en passant par Marjane Satrapi. Les nombreuses références sont classées dans un index bien pensé où les thèmes traités apparaissent aussi. Sous la forme de quatre chapitres, il étudie les aspects du cinéma français qui lui paraissent essentiels: l'importance des premiers films dans la production française (plus d'un tiers), l'émergence d'un cinéma des corps, l'analyse des films d'auteurs qui utilisent les recettes du cinéma commercial appelés "pop-art cinema " et les femmes cinéastes.

6. Nathalie Heinich, Le Triple Jeu de l'art contemporain: sociologie des arts plastiques, Paris, Éditions de Minuit, 1998, p. 96. 